\title{
Théorème de Noether stochastique
}

\section{Jacky CRESSON et Sébastien DARSES}

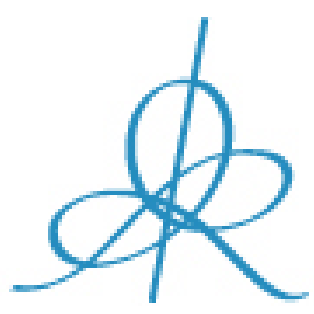

Institut des Hautes Études Scientifiques

35 , route de Chartres

91440 - Bures-sur-Yvette (France)

Mai 2006

IHES/M/06/25 


\title{
THÉORĖME DE NOETHER STOCHASTIQUE
}

\author{
par \\ Jacky CRESSON \& Sébastien DARSES
}

\begin{abstract}
Résumé. - Dans le cadre du plongement stochastique des systèmes lagrangiens [3], on définit un calcul des variations sur les processus. On démontre un théorème de Noether basé sur la notion de suspension stochastique d'un groupe à un paramètre de difféomorphismes.
\end{abstract}

\begin{abstract}
The stochastic Noether theorem). - In the framework of the stochastic embedding of lagrangian systems [3], we define a calculus of variations on stochastic processes. We prove a Noether theorem based on stochastic suspensions of one-parameter groups of diffeomorphisms.
\end{abstract}

\section{Introduction}

La procédure de plongement stochastique, définie dans [3], permet d'associer à des fonctionnelles et des équations différentielles ordinaires leurs analogues stochastiques. Pour les systèmes lagrangiens, on obtient une dynamique contrôlée par une équation d'Euler-Lagrange stochastique [3]. Que reste-t-il des propriétés de la dynamique initiale? Par exemple, dans le cas déterministe, l'invariance d'un lagrangien par un groupe de symétries induit l'existence d'une intégrale première du mouvement $(c f[1] \mathrm{p} .88)$ : c'est le contenu du théorème de Noether.

Dans cette note, on démontre un analogue stochastique du théorème de Noether. La démonstration repose sur la définition des suspensions stochastiques des groupes à un paramètre de difféomorphismes et d'un calcul des variations sur les processus stochastiques. On définit à cette occasion une notion originale d'intégrale première stochastique. 


\section{Rappels et notations}

On note $I:=] a, b[$ où $a<b$ et $J:=[a, b]$ l'adhérence de $I$ dans $\mathbb{R}$. Soit $\mathbb{K}$ un corps et $d \in \mathbb{N}^{*}$. On se donne un espace probabilisé $(\Omega, \mathcal{A}, P)$ sur lequel existent une famille croissante de tribus $\mathcal{P}:=\left(\mathcal{P}_{t}\right)_{t \in J}$ et une famille décroissante de tribus $\mathcal{F}:=\left(\mathcal{F}_{t}\right)_{t \in J}$.

Définition 2.1. - On note $\mathcal{C}_{\mathbb{K}}^{1}(J)$ l'ensemble des processus $X$ définis sur $J \times$ $\Omega$, à valeurs dans $\mathbb{K}^{d}$ et tels que : $X$ soit $\mathcal{P}$ et $\mathcal{F}$ adapté, pour tout $t \in J$ $X_{t} \in L^{2}(\Omega)$, l'application $t \rightarrow X_{t}$ de $J$ dans $L^{2}(\Omega)$ est continue, pour tout $t \in I$ les quantités

$$
D X_{t}=\lim _{h \rightarrow 0^{+}} h^{-1} E\left[X_{t+h}-X_{t} \mid \mathcal{P}_{t}\right]
$$

et

$$
D_{*} X_{t}=\lim _{h \rightarrow 0^{+}} h^{-1} E\left[X_{t}-X_{t-h} \mid \mathcal{F}_{t}\right],
$$

existent dans $L^{2}(\Omega)$, et enfin les applications $t \rightarrow D X_{t}$ et $t \rightarrow D_{*} X_{t}$ sont continues de $I$ dans $L^{2}(\Omega)$.

Le complété de $\mathcal{C}_{\mathbb{K}}^{1}(J)$ pour la norme

$$
\|X\|=\sup _{t \in I}\left(\left\|X_{t}\right\|_{L^{2}(\Omega)}+\left\|D X_{t}\right\|_{L^{2}(\Omega)}+\left\|D_{*} X(t)\right\|_{L^{2}(\Omega)}\right),
$$

est encore noté $\mathcal{C}_{\mathbb{K}}^{1}(J)$, et simplement $\mathcal{C}^{1}(J)$ quand $\mathbb{K}=\mathbb{R}$.

On note $\mathcal{D}$ la dérivée stochastique introduite dans ([3] Lemme 1.2) et définie par

$$
\mathcal{D}=\frac{D+D_{*}}{2}+i \frac{D-D_{*}}{2} .
$$

On rapelle que $\mathcal{D}$ est étendu par $\mathbb{C}$-linéarité aux processus complexes et on note

$$
\overline{\mathcal{D}}=\frac{D+D_{*}}{2}-i \frac{D-D_{*}}{2} .
$$

On note $\Lambda$ l'espace des diffusions défini dans ([3] Définition 3.1) et $\Lambda_{\mathbb{C}}$ les processus à valeurs complexes de partie réelle et imaginaire dans $\Lambda$. On montre que l'on peut calculer les dérivées $\mathcal{D}$ et $\mathcal{D}^{2}$ sur des éléments de $\Lambda$ ([2] p.26).

Théorème 2.2. - Soit $X \in \Lambda$ solution d'une EDS

$$
d X_{t}=b\left(t, X_{t}\right) d t+\sigma\left(t, X_{t}\right) d W_{t}
$$


et $f \in C^{1,2}\left(I \times \mathbb{R}^{d}\right)$ telle que $\partial_{t} f, \nabla f$ and $\partial_{i j} f$ sont bornées. En posant $a^{i j}=$ $\left(\sigma \sigma^{*}\right)^{i j}$, on obtient

$$
\mathcal{D} f\left(t, X_{t}\right)=\left(\partial_{t} f+\mathcal{D} X_{t} \cdot \nabla f+\frac{i}{2} \sum_{k, j} a^{k j} \partial_{k j} f\right)\left(t, X_{t}\right) .
$$

D'autre part, on généralise avec l'opérateur $\mathcal{D}$, la "loi produit" donnée par Nelson dans [4] p.80:

Lemme 2.3. - Soit $X, Y \in \Lambda_{\mathbb{C}}$. Alors $E\left[\mathcal{D} X_{t} \cdot Y_{t}+X_{t} \cdot \overline{\mathcal{D}} Y_{t}\right]=\frac{d}{d t} E\left[X_{t} \cdot Y_{t}\right]$.

La démonstration est une conséquence immédiate de la forme de l'opérateur $\mathcal{D}$ et du fait que $\Lambda$ est un sous-espace de la classe $S(\mathcal{F}, \mathcal{G})([8]$ p.226) pour laquelle W. Zheng et P-A. Meyer démontrent la loi produit de Nelson ( $c f$ [8] Théorème I.2 p.227).

\section{Calcul des variations stochastique dynamique}

On définit un calcul des variations à partir de fonctionnelles lagrangiennes définies sur $\mathcal{C}^{1}(J)$. Il repose sur la dérivée stochastique dynamique $\mathcal{D}$. Le calcul des variations stochastiques de Malliavin quant à lui repose sur une notion de différentiation suivant le paramètre aléatoire $\omega \in \Omega$ (voir [5] p.24).

On dit qu'un lagrangien $L$ est admissible si la fonction $L(x, v)$ est définie sur $\mathbb{R}^{d} \times \mathbb{C}^{d}, C^{1}$ en $x$ et holomorphe en $v$, et est réelle quand $v$ est réel.

Le lagrangian $L$ est dit naturel s'il s'écrit

$$
L(x, v)=q(v)-U(x)
$$

où $q$ est une forme quadratique sur $\mathbb{C}^{d}$ et $U$ un potentiel de classe $\mathcal{C}^{1}$ sur $\mathbb{R}^{d}$.

La fonctionnelle $F_{J}$ associée à $L$ est définie à partir du plongement de la fonction lagrangienne $L\left(x(t), x^{\prime}(t)\right)$ :

$$
F_{J}:\left\{\begin{array}{ccc}
\Xi \subset \mathcal{C}^{1}(J) & \rightarrow & \mathbb{C} \\
X & \mapsto & E\left[\int_{J} L\left(X_{t}, \mathcal{D} X_{t}\right) d t\right]
\end{array}\right.
$$

avec

$$
\Xi=\left\{X \in \mathcal{C}^{1}(J), E\left[\int_{J}\left|L\left(X_{t}, \mathcal{D} X_{t}\right)\right| d t\right]<\infty\right\} .
$$

On en déduit une notion de différentiabilité liée à $F_{J}$. On appelle $\Gamma$-variation de $X \in \mathcal{C}^{1}(J)$, un processus de la forme $X+Z$ où $Z \in \Gamma$ et on pose

$$
\Gamma_{\Xi}=\{Z \in \Gamma, \forall X \in \Xi, Z+X \in \Xi\} .
$$


Définition 3.1. - Si L est un lagrangien admissible et $F_{J}$ la fonctionnelle associée, $F_{J}$ est dite $\Gamma$-différentiable en un processus $X \in \Xi$ si pour tout $Z \in$ $\Gamma_{\Xi}$

$$
F_{J}(X+Z)-F_{J}(X)=d F_{J}(X, Z)+R_{X}(Z),
$$

où $d F_{J}(X, Z)$ est une fonctionnelle linéaire en $Z$ et $R_{X}(Z)=o(\|Z\|)$. De plus, $X$ est dit $\Gamma$-stationnaire si pour tout $Z \in \Gamma_{\Xi}, d F_{J}(X, Z)=0$.

La définition de la dérivée stochastique dynamique contraint l'espace des variations (i.e. $Z \in \Gamma_{\Xi}$ ). De la même manière, la définition de la dérivée de Malliavin détermine l'espace des variations de Cameron-Martin ([5] p.25).

Théorème 3.2. - Un processus $X$ est $\mathrm{C}^{1}(J)$-stationnaire pour $F_{J}$ si et seulement c'est une solution de l'équation

$$
\left(\partial_{x} L-\overline{\mathcal{D}} \partial_{v} L\right)\left(X_{u}, \mathcal{D} X_{u}\right)=0
$$

sur $I$.

On renvoie à ([2] Chapitre 7) pour la démonstration.

\section{Théorème de Noether stochastique}

Soit $\Pi$ l'ensemble des processus définis sur $J \times \Omega$, et $C^{k}(J)$ l'ensemble des fonctions de classe $C^{k}$ de $J$ dans $\mathbb{R}^{d}, k \in \mathbb{N}$.

Définition 4.1. - Soit $\phi: \mathbb{R}^{d} \rightarrow \mathbb{R}^{d}$ un difféomorphisme. La suspension stochastique de $\phi$ est l'application $\Phi: \Pi \rightarrow \Pi$ définie par

$$
\forall X \in \Pi, \Phi(X)_{t}(\omega)=\phi\left(X_{t}(\omega)\right) .
$$

Dans la suite on notera indifféremment le difféomorphisme et sa suspension.

De plus un groupe à un paramètre de transformations $\Phi_{s}: \Upsilon \rightarrow \Upsilon, s \in \mathbb{R}$, où $\Upsilon \subset \Pi$, est appelé un groupe $\phi$-suspendu agissant sur $\Upsilon$ s'il existe un groupe à un paramètre de difféomorphisme $\phi_{s}: \mathbb{R}^{d} \rightarrow \mathbb{R}^{d}, s \in \mathbb{R}$, tel que pour tout $s \in \mathbb{R}$, $\Phi_{s}$ soit une suspension stochastique de $\phi_{s}$, et pour tout $X \in \Upsilon, \Phi_{s}(X) \in \Upsilon$.

Définition 4.2. - Un groupe à un paramètre de difféomorphismes est dit admissible si $\Phi=\left\{\phi_{s}\right\}_{s \in \mathbb{R}}$ est un groupe à un paramètre de $C^{2}$-diffeomorphismes sur $\mathbb{R}^{d}$ tel que $(s, x) \mapsto \partial_{x} \phi_{s}(x)$ est de classe $C^{2}$ et tel que la formule (7) reste vrai pour toute fonction $\phi_{s}$ du groupe.

On étudie le comportement des suspensions par rapport à l'espace $\Lambda$. Grâce au lemme de Schwarz et au théorème 2.2, on montre : 
Lemme 4.3. - Soit $\Phi=\left(\phi_{s}\right)_{s \in \mathbb{R}}$ une suspension stochastique d'un groupe admissible à un paramètre de difféomorphismes. Alors pour tout $X \in \Lambda$, et pour tout $(t, s) \in I \times \mathbb{R}$ l'application $s \mapsto \mathcal{D}\left(\Phi_{s} X\right)_{t}$ est de classe $C^{1}$ p.s. et

$$
\partial_{s}\left[\mathcal{D}\left(\phi_{s}(X)\right)\right]=\mathcal{D}\left[\partial_{s} \phi_{s}(X)\right] \text { p.s.. }
$$

La notion d'invariance par une suspension stochastique est :

Définition 4.4. - Soit $\Phi=\left(\phi_{s}\right)_{s \in \mathbb{R}}$ une suspension stochastique d'un groupe admissible à un paramètre de difféomorphismes et $L: \mathcal{C}^{1}(I) \rightarrow \mathcal{C}_{\mathbb{C}}^{1}(I)$. La fonctionnelle $L$ est invariante sous $\Phi$ si pour tout $s \in \mathbb{R}$ et $X \in \mathrm{C}^{1}(J)$,

$$
L\left(\phi_{s} X, \mathcal{D}\left(\phi_{s}(X)\right)\right)=L(X, \mathcal{D} X) .
$$

La définition d'invariance utilisée par M. Thieullen et J-C. Zambrini ([6] p.313) peut se formuler à l'aide des suspensions stochastiques de groupes à un paramètre de difféomorphismes.

Exemple. On considère le lagrangien étendu du problème des deux corps dans $\mathbb{R}^{3}$, i.e. $\mathcal{L}(x, z)=q(z)-\frac{1}{|x|}$ où pour tout $x \in \mathbb{R}^{3},|x|^{2}=x_{1}^{2}+x_{2}^{2}+x_{3}^{2}$ et tout $z \in \mathbb{C}^{3}, q(z)=z_{1}^{2}+z_{2}^{2}+z_{3}^{2}$.

Lemme 4.5. - Le lagrangien $\mathcal{L}$ défini sur $\mathbb{R}^{3} \backslash\{0\} \times \mathbb{C}^{3}$ est invariant par la suspension stochastique des rotations $\phi_{\theta, k}$ d'axes $e_{k}$ (base canonique) et d'angle $\theta, k=1,2,3$.

Démonstration. - Comme $\phi_{\theta, k}$ est une matrice dont les coefficients ne dépendent pas de $t$, on a $\mathcal{D}_{\mu}\left[\phi_{\theta, k}(X)\right]=\phi_{\theta, k}\left[\mathcal{D}_{\mu} X\right]$ où $\phi_{\theta, k}$ est trivialement étendu à $\mathbb{C}^{3}$. Un calcul simple donne pour tout $z \in \mathbb{C}^{3}, q\left(\phi_{\theta, k}(z)\right)=q(z)$. On déduit alors que $\mathcal{L}\left(\phi_{\theta, k} X, \mathcal{D}\left(\phi_{\theta, k} X\right)\right)=\mathcal{L}(X, \mathcal{D} X)$.

Théorème 4.6 (Noether stochastique). - Soit $F_{J}$ la fonctionnelle définie sur $\Xi \cap \Lambda$ par (9), où $L$ est un lagrangien admissible invariant sous le groupe admissible à un paramètre de difféomorphisme $\Phi=\left(\phi_{s}\right)_{s \in \mathbb{R}}$. Soit $X^{0} \in \Xi \cap \Lambda$ un point $\mathrm{C}^{1}(J)$-stationnaire de $F_{J}$. On pose $Y_{t}(s)=\left(\Phi_{s} X^{0}\right)_{t}$. Alors

$$
\frac{d}{d t} E\left[\partial_{v} L\left(X^{0}, \mathcal{D} X_{t}^{0}\right) \cdot \frac{\partial Y_{t}}{\partial s}(0)\right]=0 .
$$

Démonstration. - On pose $V_{t}(s)=\left(Y_{t}(s), \mathcal{D} Y_{t}(s)\right)$. Comme $L$ est invariant sous $\Phi=\left\{\phi_{s}\right\}_{s \in \mathbb{R}}$, on a $\frac{\partial}{\partial s} L\left(V_{t}(s)\right)=0 \quad$ (p.s.). Comme pour tout $t \in J$ et tout $\omega \in \Omega, Y_{t}(\cdot)(\omega) \in C^{1}(\mathbb{R})$ et $\mathcal{D} Y_{t}(\cdot)(\omega) \in C^{1}(\mathbb{R})$, on obtient

$$
\partial_{x} L\left(V_{t}(s)\right) \cdot \frac{\partial Y_{t}}{\partial s}+\partial_{v} L\left(V_{t}(s)\right) \cdot \frac{\partial \mathcal{D} Y_{t}}{\partial s}=0 \quad \text { (p.s.). }
$$


En utilisant le Lemme 4.3, cette équation est équivalente à

$$
\left.\partial_{x} L\left(V_{t}(s)\right) \cdot \frac{\partial Y_{t}}{\partial s}+\partial_{v} L\left(V_{t}(s)\right) \cdot \mathcal{D}\left(\frac{\partial Y_{t}}{\partial s}\right)=0 \quad \text { (p.s. }\right) .
$$

Comme $X^{0}=Y(0)$ est un point $\mathcal{C}^{1}(J)$-stationaire de $F_{J}$, on a

$$
\partial_{x} L\left(V_{t}(0)\right)=\overline{\mathcal{D}}\left[\partial_{v} L\left(V_{t}(0)\right)\right] .
$$

On en déduit alors

$$
\left.\overline{\mathcal{D}}\left[\partial_{v} L\left(V_{t}(0)\right)\right] \cdot \frac{\partial Y_{t}}{\partial s}(0)+\partial_{v} L\left(V_{t}(0)\right) \cdot \mathcal{D}\left(\frac{\partial Y_{t}}{\partial s}(0)\right)=0 \quad \text { (p.s. }\right) .
$$

D'où

$$
E\left[\overline{\mathcal{D}}\left[\partial_{v} L\left(V_{t}(0)\right)\right] \cdot \frac{\partial Y}{\partial s}(0)+\partial_{v} L\left(V_{t}(0)\right) \cdot \mathcal{D}\left(\frac{\partial Y_{t}}{\partial s}(0)\right)\right]=0 .
$$

Avec le Lemme 2.3, il vient

$$
\frac{d}{d t} E\left[\partial_{v} L\left(V_{t}(0)\right) \cdot \frac{\partial Y_{t}}{\partial s}(0)\right]=0
$$

Ce résultat contient le théorème de Noether classique via l'injection $\iota: C^{1}(J) \rightarrow \Lambda$ définie par $(\iota x)_{t}(\omega):=x(t)$ pour tous $x \in C^{1}(J), t \in \mathbb{R}, \omega \in \Omega$.

La notion d'intégrale première qui découle de ce théorème est :

Définition 4.7. - Soit $L$ un lagrangien admissible. Une fonctionnelle $\mathcal{I}$ : $L^{2}(\Omega) \rightarrow \mathbb{C}$ est une intégrale première d'un processus $\Gamma$-stationnaire de la fonctionnelle $F_{J}$ si $\frac{d}{d t}\left[\mathcal{I}\left(X_{t}\right)\right]=0$.

Cette définition est naturelle en ce sens que l'analogue d'une courbe classique $J \rightarrow \mathbb{R}^{d}$ (une fonction) est ici une courbe $J \rightarrow L^{2}(\Omega)$ (un processus). Pour des courbes annulant la différentielle de la fonctionnelle $F_{J}$, on cherche les constantes de ce "mouvement dans $L^{2}(\Omega)$ ".

Une alternative à cette définition consiste, pour un processus stationnaire $\left(X_{t}\right)_{i \in J}$ donné, à définir une constante non sur l'objet processus $t \rightarrow X_{t}$, mais sur l'ensemble de ces trajectoires $t \mapsto X_{t}(\omega)$. L'intégrale première est alors vue comme un processus constant, dépendant du processus critique. Ce point de vue est développé par M. Thieullen et J-C. Zambrini dans [6] : les auteurs montrent qu'à partir d'un point critique pour leur fonctionnelle d'action on peut construire un processus conditionnellement constant, i.e une martingale. 


\section{Références}

[1] V.I. Arnold, Mathematical Methods of Classical Mechanics, 2d edition, Springer, 1989.

[2] J. Cresson, S. Darses, Stochastic embedding of dynamical systems, arXiv :math.PR/0509713, 2005, 112.p.

[3] J. Cresson, S. Darses, Plongement stochastique des systèmes lagangiens, C.R. Acad. Sci. Paris, Ser. I 342, 333-336, (2006).

[4] E. Nelson, Dynamical theories of Brownian motion, Second edition, Princeton University Press, 2001.

[5] D. Nualart, The Malliavin Calculus and Related Topics, Springer Verlag (1996).

[6] M. Thieullen, J.C. Zambrini, Probability and quantum symmetries I. The theorem of Noether in Schrödinger 's euclidean mechanics, Ann. Inst. Henri Poincaré, Physique théorique, Vol 67, $\mathrm{n}^{\circ} 3$ p.297-338 (1997).

[7] K. Yasue, Stochastic calculus of variations, Journal of functional Analysis 41, 327-340 (1981).

[8] W.A. Zheng, P.A. Meyer, Quelques résultats de "mécanique stochastique", Séminaire de Probabilités XVIII, 223-244 (1982/83).

\footnotetext{
JACKY CRESSON • E-mail : jacky.cresson@univ-pau.fr et cresson@ihes.fr, Université de Pau et des Pays de l'Adour et Institut des Hautes Études Scientifiques DARSES - E-mail : darses@math.univ-fcomte.fr, Laboratoire de Mathématiques de Besançon, CNRS UMR 6623, Université de Franche-Comté
} 